\title{
Uso de modelos de volatilidad estocástica para valoración de riesgo cambiario
}

\author{
Use of stochastic volatility models for exchange rate risk valuation
}

José Fernando Zea Castrd国

jfzeac@gmail.com

\begin{abstract}
Resumen
En la literatura técnica y en las aplicaciones de finanzas es muy importante cuantificar el riesgo de mercado. En el caso de tasas de cambio que usan series de tiempo diarias es común el uso de la estrategia de RiskMetrics ${ }^{T M}$ junto con los modelos GARCH. Varios autores en diferentes contextos han destacado las falencias del uso de modelos GARCH. Este trabajo aborda el cálculo de Valor en Riesgo con el uso de modelos de volatilidad estocástica (SV). Luego de mencionar las ventajas y desventajas que ofrecen estos modelos frente a los GARCH, presenta los resultados de evaluar el riesgo de un portafolio que contiene tasas de cambio diarias de Peso/Dólar, Euro/Dólar, Yen/Dólar y Libra/Dólar. El ejercicio muestra resultados que favorecen el uso de modelos de SV.
\end{abstract}

Palabras clave: Modelos GARCH, modelos de volatilidad estocástica, valor en riesgo.

\begin{abstract}
Market risk valuation is very important not only for financial theoreticians but for the applied job. When using daily time series on exchange rates, the use of RiskMetrics $^{T M}$ strategy, in conjunction with GARCH models, is common place. Many authors, in several contexts, have pointed out the inconvenience in using GARCH models. In this paper the use of Stochastic Volatility (SV) models in risk valuation is considered. We describe the main advantages and disadvantages of SV models against GARCH models. We present results of market risk valuation using SV for a portfolio that contains daily returns of Euro/Dollar, Yen/Dollar, UK\$/Dollar and exchange rates. Our results suggest that the SV model produce more reliable estimations.
\end{abstract}

Key words: GARCH models, stochastic volatility models, value at risk.

${ }^{a}$ Asesor estadístico. Secretaría de Desarrollo Económico. 


\section{Introducción}

Una de las principales preocupaciones de los inversionistas y de los llamados agentes que participan en el mercado financiero es tener un conocimiento aproximado del riesgo que presentan las inversiones que realizan. El riesgo desde un amplio punto de vista se refiere a una alta probabilidad de ocurrencia de una calamidad o evento negativo. Desde un punto de vista financiero el riesgo tiene una connotación distinta, se entiende como la incertidumbre en la obtención de un resultado en las diferentes actividades financieras. Esta definición abarca no solo pérdidas y malos resultados sino también las potenciales ganancias que el mercado ofrece. Un concepto muy importante que surge dentro de las series de tiempo es el de volatilidad, ya que una forma simple de medir el riesgo de un activo es a través de la volatilidad de sus retornos; cuando un activo tiene alta volatilidad, su resultado futuro presenta una mayor incertidumbre. Las ganancias y pérdidas que se obtienen al participar en el mercado financiero involucran una alta incertidumbre asociada a altas volatilidades. Varios intentos se han hecho para incorporar la volatilidad en los modelos de series de tiempo. Existen, además del tradicional suavizamiento exponencial (EWMA), principalmente dos enfoques: en primer lugar los modelos ARCH con sus modificaciones y generalizaciones, y en segundo lugar los modelos de volatilidad estocástica (Tsay 2002). Un adecuado modelado y pronóstico de la volatilidad en estos modelos permite hacer predicciones de los retornos financieros. Se quiere evaluar la idoneidad de los modelos de volatilidad estocástica para el mercado financiero colombiano.

Este documento está organizado como sigue: en la sección 2 se discuten los conceptos de la representaciones Espacio de Estados y el Filtro de Kalman; en la sección 3 se presentan algunos resultados de modelos para la heterocedasticidad; la sección 4 contiene ideas sobre cálculo del Valor en Riesgo; en la sección 5 se describen los ejercicios y presentan los resultados de los mismos; y finalmente en la sección 6 se resumen, concluyen y recomiendan algunas extensiones.

\section{Modelos de estados}

Los modelos de estados (State Space) o modelos lineales dinámicos (MLD) están conformados, en su forma básica, por un vector autorregresivo de orden uno,

$$
\mathbf{x}_{t}=\Phi \mathbf{x}_{t-1}+\mathbf{w}_{t},
$$

La ecuación (11) es conocida como la ecuación de estado y determina las reglas para la generación de los $p \times 1$ estados, $\mathbf{x}_{t}$, para los puntos $t=1, \ldots, n$. Se asume que $\mathbf{w}_{t}$ son vectores $p \times 1$, normales independientes e idénticamente distribuidos con media cero y matriz de covarianzas Q. En el MLD, se asume que el proceso empieza con un vector normal $\mathbf{x}_{0}$ que tiene media $\mu_{0}$ y matriz de covarianzas $\Sigma_{0}$ de tamaño $p \times p$. En cuanto a $\Phi$ es una matriz de tamaño $p \times 1$. El MLD, añade, sin embargo, un componente adicional en el modelo al asumir que no se observa 
el vector estado $\mathbf{x}_{t}$ directamente sino mediante una transformación lineal de este con la adición de un proceso ruido blanco, es decir

$$
\mathbf{y}_{t}=A_{t} \mathbf{x}_{t}+\mathbf{v}_{t},
$$

donde $A_{t}$ es una matriz $q \times p$ la cual es conocida como la matriz de observaciones. La ecuación (2) es llamada la ecuación de observación o medida. Los datos observados están en $\mathbf{y}_{t}$, vector de tamaño $q \times 1$, el cual puede ser más grande o más pequeño que $p$, la dimensión de la serie de interés. El ruido $\mathbf{v}_{t}$ de la ecuación de observación es aditivo y se asume blanco y gaussiano con matriz de covarianzas $\mathrm{R}$ de tamaño $q \times q$. Por simplicidad se asume que $\left\{\mathbf{w}_{t}\right\}$ y $\left\{\mathbf{v}_{t}\right\}$ están incorreladas. Se puede modificar el modelo básico (11) y (2) para incluir variables exógenas. En este caso se supone que el vector de entrada $\left\{\mathbf{u}_{t}\right\}$ de tamaño $r \times 1$ y se escribe el modelo como

$$
\begin{gathered}
\mathbf{x}_{t}=\Phi \mathbf{x}_{t-1}+\Upsilon \mathbf{u}_{t}+\mathbf{w}_{t} \\
\mathbf{y}_{t}=A_{t} \mathbf{x}_{t}+\Gamma \mathbf{u}_{t}+\mathbf{v}_{t}
\end{gathered}
$$

donde $\Upsilon$ es $p \times r$ y $\Gamma$ es $q \times r$

\subsection{Filtro y predicción}

El principal objetivo del análisis que involucra a los modelos de estados definidos en (11) y (2) o por (3) y (4) será el de producir estimadores para la señal no observada $\mathbf{x}_{t}$ dados los datos $Y_{s}=\left\{\mathbf{y}_{1}, \ldots, \mathbf{y}_{s}\right\}$, al tiempo $s$. Cuando $s<t$, el problema es llamado predicción o pronóstico (prediction). Cuando $s=t$ el problema es llamado «filtrado» (filtering), y cuando $s>t$, es llamado suavizamiento (smoothing). En adición a estos resultados se quiere también una medida de precisión. La solución a este requerimiento es mediante el filtro de Kalman. Se utilizarán las siguientes definiciones:

$$
\mathbf{x}_{t}^{s}=E\left(\mathbf{x}_{t} / Y_{s}\right)
$$

y

$$
P_{t_{1}, t_{2}}=E\left\{\left(\mathbf{x}_{t_{1}}-\mathbf{x}_{t_{1}}^{s}\right)\left(\mathbf{x}_{t_{2}}-\mathbf{x}_{t_{2}}^{s}\right)^{\prime}\right\}
$$

Cuando $t_{1}=t_{2}$ (por ejemplo igual a t) en (6) , por conveniencia, se escribirá $P_{t}^{s}$.

Para obtener las ecuaciones de «filtrado» se tendrá en cuenta el supuesto gaussiano, en el caso no gaussiano se obtendrán estimadores de error mínimo cuadrático dentro la clase de estimadores lineales.

Cuando se asume que los procesos son gaussianos la ecuación (66) es también la covarianza condicional del error 


$$
P_{t_{1}, t_{2}}=E\left\{\left(\mathbf{x}_{t_{1}}-\mathbf{x}_{t_{1}}^{s}\right)\left(\mathbf{x}_{t_{2}}-\mathbf{x}_{t_{2}}^{s}\right)^{\prime} / Y_{s}\right\}
$$

A continuación se presenta el filtro de Kalman, el cual genera las ecuaciones de filtrado y pronóstico. El nombre de filtro proviene del hecho de que $\mathbf{x}_{t}^{t}$ es un filtro lineal de las observaciones $\mathbf{y}_{1}, \ldots, \mathbf{y}_{t}$; esto es, $\mathbf{x}_{t}^{t}=\sum_{s=1}^{t} B_{s} \mathbf{y}_{s}$, para una adecuada elección de las matrices $B_{s}$, de tamaño $p \times q$. La ventaja del filtro de Kalman es que especifica cómo actualizar el filtro de $\mathbf{x}_{t-1}^{t-1}$ a $\mathbf{x}_{t}^{t}$ una vez que una nueva observación $\mathbf{y}_{t}$ es obtenida, sin tener que preprocesar todos los datos $\mathbf{y}_{1}, \ldots, \mathbf{y}_{t}$.

\subsection{El filtro de Kalman}

Para los modelos de estados especificados en (3) y (4) con condiciones iniciales $\mathbf{x}_{0}^{0}=\mu_{0}$ y $P_{0}^{0}=\Sigma_{0}$, para $t=1, \ldots, n$,

$$
\begin{aligned}
& \mathbf{x}_{t}^{t-1}=\Phi \mathbf{x}_{t-1}^{t-1}+\Upsilon u_{t}, \\
& P_{t}^{t-1}=\Phi P_{t-1}^{t-1} \Phi^{\prime}+Q
\end{aligned}
$$

con

$$
\begin{gathered}
\mathbf{x}_{t}^{t}=\Phi \mathbf{x}_{t}^{t-1}+K_{t}\left(\mathbf{y}_{t}-A_{t} x_{t}^{t-1}-\Gamma u_{t}\right), \\
P_{t}^{t}=\left[I-K_{t} A_{t}\right] P_{t}^{t-1},
\end{gathered}
$$

donde

$$
K_{t}=P_{t}^{t-1} A_{t}^{\prime}\left[A_{t} P_{t}^{t-1} A_{t}^{\prime}+R\right]^{-1}
$$

es llamado la ganancia de Kalman.

La predicción para $t>n$, o fuera de muestra, es llevada a cabo mediante (8) y (9), con condiciones iniciales $\mathbf{x}_{n}^{n}$ y $P_{n}^{n}$.

\subsection{Estimación máximo-verosímil}

La estimación de los parámetros involucrada con los modelos de estados, (3) y (41), es usualmente bastante tediosa. Si se usa $\Theta=\left\{\mu_{0}, \Sigma_{0}, \Phi, Q, R, \Upsilon, \Gamma\right\}$ para representar el conjunto de parámetros que contienen los elementos de media y covarianza inicial $\mu_{0}$ y $\Sigma_{0}$, la matriz de transición $\Phi$ y las matrices de covarianzas del estado y la observación Q y R y la matriz de coeficientes de entrada, $\Upsilon$ y $\Gamma$. Se usa la verosimilitud bajo el supuesto de que el estado inicial sigue una distribución normal, $\mathbf{x}_{0} \sim \mathrm{N}\left(\mu_{0}, \Sigma_{0}\right)$, y los errores $\mathbf{w}_{1}, \ldots, \mathbf{w}_{n} \mathrm{y} \mathbf{v}_{1}, \ldots, \mathbf{v}_{n}$ son normales conjuntos y variables de vectores no correlacionados. Se asume por simplicidad 
que $\left\{w_{t}\right\}$ y $\left\{v_{t}\right\}$ están no correlacionados. La verosimilitud es calculada usando las innovaciones $\epsilon_{1}, \epsilon_{2}, \ldots, \epsilon_{n}$, definidas por

$$
\epsilon_{t}=\mathbf{y}_{t}-A_{t} \mathbf{x}_{t}^{t-1}-\Gamma \mathbf{u}_{t}
$$

Las innovaciones forman la función de verosimilitud, las innovaciones tienen media y matrices de covarianzas dadas por $E\left(\epsilon_{t}\right)=0$, y $\Sigma_{t}=\operatorname{Var}\left(\epsilon_{t}\right)=A_{t} P_{t}^{t-1} A_{t}^{\prime}+R$, respectivamente.

Como propiedad adicional se tiene que $E\left[\epsilon_{t} \mathbf{y}_{s}^{\prime}\right]=0$ para $s<t$ lo cual es resultado del hecho que la secuencia de las innovaciones es un proceso gaussiano, lo cual implica que las innovaciones son independientes de las observaciones pasadas.

Ignorando la constante, se puede escribir la función de verosimilitud, $L_{y}(\Theta)$, como

$$
-\ln L_{Y}(\Theta)=\frac{1}{2} \sum_{t=1}^{n} \log \left|\Sigma_{t}(\Theta)\right|+\frac{1}{2} \sum_{t=1}^{n} \epsilon_{t}(\Theta)^{\prime} \Sigma_{t}^{-1} \epsilon_{t}(\Theta)
$$

donde se ha enfatizado la dependencia de las innovaciones en los parámetros $\Theta$. Esta es una función no lineal de los parámetros desconocidos y tiene un alto grado de complejidad. El procedimiento usual es ajustar $\mathbf{x}_{0}$ y luego desarrollar un conjunto de recurrencias sobre el logaritmo de la función de verosimilitud, encontrar su máximo y sus primeras dos derivadas (véase por ejemplo Shumway \& Stoffer (2006)). El algoritmo Newton-Raphson puede ser usado sucesivamente para actualizar los parámetros hasta que el negativo del logaritmo de la función de verosimilitud sea minimizado. Los pasos del algoritmo de Newton son los siguientes.

1. Seleccione valores iniciales para los parámetros por decir $\Theta^{0}$.

2. Calcule el filtro de Kalman, usando los valores iniciales de los parámetros, $\Theta^{0}$, para obtener un conjunto de innovaciones y covarianzas en los errores, es decir, $\left\{\epsilon_{t}^{0}=1, \ldots, n\right\}$ y $\left\{\Sigma_{t}^{0}=1, \ldots, n\right\}$

3. Realice una iteración del procedimiento Newton-Raphson con $-\ln L_{\Theta}$ para obtener un nuevo conjunto de estimaciones, por decir $\Theta^{1}$.

4. En la iteración $\mathbf{j},(j=1,2, \ldots)$, repetir el paso 2 usando $\Theta^{(j)}$ en lugar de $\Theta^{(j-1)}$ para obtener un nuevo conjunto de valores innovación $\left\{\epsilon_{t}^{j} ; t=1, \ldots, n\right\}$ y $\left\{\Sigma_{t}^{j} ; t=1, \ldots, n\right\}$. Luego repetir el paso (3) para obtener una nueva estimación $\Theta^{j+1}$. El algoritmo se detiene cuando el valor de la verosimilitud se estabiliza, por ejemplo, cuando los valores de $\Theta^{j+1}$ no difieren de $\Theta^{j}$, o cuando $L_{Y}\left(\Theta^{(j+1)}\right)$ difiere de $L_{Y}\left(\Theta^{(j)}\right)$ por alguna cantidad predeterminada pero que sea muy pequeña.

\subsection{Modelo Lineal con Switching}

El problema de modelar cambios de régimen para los valores de series de tiempo ha sido de interés en muchos campos. Algunos autores como Hamilton (1989), McCulloch \& Tsay (1993) y Tsay (2002), han explorado varios modelos para esto. 
Para empezar se retoma (11) y (2) con los mismos supuestos, Shumway \& Stoffer (2006) tratan a la matriz $A_{t}$ en (2) como cambiante en el tiempo. Para incorporar una estructura de switching razonable para la matriz de medida $A_{t}$ en el Modelo Lineal Dinámico, se asume que $m$ posibles configuraciones $M_{1}, M_{2}, \ldots, M_{m}$ para $A_{t}$, cada una con las siguientes probabilidades,

$$
\pi_{j}(t)=\operatorname{Pr}\left(A_{t}=M_{j}\right)
$$

para $j=1, \ldots, m$ y $t=1,2, \ldots, n$. Una información importante sobre el estado corriente del proceso de medida es dada por las probabilidades de filtrado de estar en el estado $j$, definido como las probabilidades condicionales

$$
\pi_{j}(t / t)=\operatorname{Pr}\left(A_{t}=M_{j} / Y_{t}\right)
$$

la cual es una función del tiempo. Con la notación $Y_{s}=\left\{\mathbf{y}_{1}, \ldots, \mathbf{y}_{s}\right\}$. Las probabilidades de filtro dan las estimaciones variantes en el tiempo de la probabilidad de estar en el estado $j$ dados los datos hasta el tiempo $t$. Es importante obtener estimadores de las probabilidades de configuración, $\pi_{j}(t / t)$, los predictores y los estimadores de los filtros del estado, $\mathbf{x}_{t}^{t-1} \mathrm{y} \mathbf{x}_{t}^{t} \mathrm{y}$ las correspondientes matrices de covarianzas de los errores $P_{t}^{t-1}$ y $P_{t}^{t}$. Por supuesto, el predictor y los estimadores del filtro dependerán de los parámetros $\Theta$, del modelo lineal dinámico. En muchas ocasiones los parámetros serán desconocidos y deberán ser estimados. Enseguida se presentan las recursiones de los filtros asociados con el estado $\mathbf{x}_{t}$, y el proceso de switching, $A_{t}$. Como se discutió anteriormente, los filtros son una parte esencial del procedimiento de máxima verosimilitud. Los predictores $\mathbf{x}_{t}^{t-1}=E\left(x_{t} / Y_{t-1}\right)$, y los filtros $x_{t}^{t}=E\left(x_{t} / Y_{t}\right)$, y sus matrices de varianzas y covarianzas asociadas al error, $P_{t}^{t-1}$ y $P_{t}^{t}$, están dadas por

$$
\begin{gathered}
\mathbf{x}_{t}^{t-1}=\Phi \mathbf{x}_{t-1}^{t-1}, \\
P_{t}^{t-1}=\Phi P_{t-1}^{t-1} \Phi^{\prime}+Q \\
\mathbf{x}_{t}^{t}=\mathbf{x}_{t}^{t-1}+\sum_{j=1}^{m} \pi_{j}(t / t) K_{t j} \epsilon_{t j}, \\
P_{t}^{t}=\sum_{j=1}^{m} \pi_{j}(t / t)\left(I-K_{t j} M_{j}\right) P_{t}^{t-1} \\
K_{t j}=P_{t}^{t-1} M_{j}^{\prime} \Sigma_{t j}^{-1}
\end{gathered}
$$

donde los valores de las innovaciones en (19) y (20) son:

$$
\epsilon_{t j}=y_{t}-M_{j} \mathbf{x}_{t}^{t-1}
$$




$$
\Sigma_{t j}=M_{j} P_{t}^{t-1} M_{j}^{\prime}+R
$$

para $j=1, \ldots, m$.

El filtro para $\pi_{j}(t / t)$. Se denota con $f_{j}(t / t-1)$ la densidad condicional de $\mathbf{y}_{t}$ dado el pasado $\mathbf{y}_{1}, \ldots, \mathbf{y}_{t-1}$, y $A_{t}=M_{j}$, para $j=1, \ldots m$. Luego

$$
\pi_{j}(t / t)=\frac{\pi_{j}(t) f_{j}(t / t-1)}{\sum_{k=1}^{m} \pi_{k}(t) f_{k}(t / t-1)}
$$

donde se asume que la distribución $\pi_{j}(t)$, para $j=1, \ldots m$, ha sido especificada al observar $y_{1}, \ldots, \mathbf{y}_{t}$. Si el investigador no tiene razón para preferir un estado sobre otro en el tiempo $t$ se escogerán valores a priori uniformes $\pi_{j}(t)=m^{-1}$, para $j=1, \ldots, m$ la computación para el cálculo $f_{j}(t / t-1)$ es intensiva, por tanto para las aplicaciones practicas de switching se utilizarán diversas aproximaciones (Shumway \& Stoffer 2006).

\section{Modelos de heterocedasticidad condicionada}

Tsay (2002) se refiere a volatilidad como la varianza condicionada de una serie, la cual puede ser por ejemplo el retorno de un activo. Una característica especial de la volatilidad es que no es directamente observable de los retornos porque usualmente hay una observación en un día de mercado. La no observabilidad de la serie hace que sea difícil evaluar el desempeño del pronóstico de un modelo de heterocedasticidad condicional. Aunque la volatilidad no es directamente observable, tiene ciertas características que son comúnmente vistas en los retornos de los activos: primero existen agrupamientos de volatilidad (la volatilidad puede ser alta en algunos períodos de tiempo y baja durante otros); segundo, la volatilidad evoluciona a través del tiempo de una manera continua, esto es, los saltos bruscos de volatilidad son raros; tercero, la volatilidad no diverge a infinito, esto significa que la volatilidad varía dentro de un rango fijo. Estadísticamente hablando esto significa que la volatilidad es usualmente estacionaria.

Sea $r_{t}$ el retorno al tiempo $t$, la idea detrás de los estudios de volatilidad es que las series $\left\{r_{t}\right\}$ son o serialmente incorreladas o tienen correlaciones con un orden pequeño; sin embargo, estas son dependientes ya que usualmente los cuadrados de los retornos muestran correlaciones seriales significativas. La media y la varianza condicional de $r_{t}$ dado $Y_{t-1}$, son,

$$
\begin{gathered}
\mu_{t}=E\left\{r_{t} / Y_{t-1}\right\}, \\
\sigma_{t}^{2}=\operatorname{Var}\left(r_{t} / Y_{t-1}\right)=E\left[\left(r_{t}-\mu_{t}\right)^{2} / Y_{t-1}\right],
\end{gathered}
$$

donde $Y_{t-1}$ denota la información disponible hasta el tiempo $t-1$. Como ya se había mencionado, la dependencia serial de un retorno de un activo es débil, si es 
que existe, por lo tanto el modelo para $\mu_{t}$ en (25) debe ser simple, y se asume que $r_{t}$ sigue un modelo de serie de tiempo $\operatorname{ARMA}(\mathrm{p}, \mathrm{q})$. En otras palabras,

$$
\begin{gathered}
r_{t}=\mu_{t}+a_{t} \\
\mu_{t}=\phi_{0}+\sum_{i=1}^{p} \phi_{i} r_{t-i}-\sum_{i=1}^{q} \theta_{i} a_{t-i},
\end{gathered}
$$

donde $p$ y $q$ son enteros no negativos. En los modelos de heterocedasticidad estocástica se va a tener que:

$$
\sigma_{t}^{2}=\operatorname{Var}\left(r_{t} / Y_{t-1}\right)=\operatorname{Var}\left(a_{t} / Y_{t-1}\right)
$$

La manera en la cual $\sigma_{t}^{2}$ se comporta en el tiempo distinguirá un modelo de volatilidad de otro.

Los modelos de volatilidad pueden ser clasificados en dos categorías. Los de la primera categoría son aquellos que usan una función exacta para gobernar la evolución de $\sigma_{t}^{2}$, mientras que en la segunda categoría se usa una ecuación estocástica para describir $\sigma_{t}^{2}$. Los modelos GARCH pertenecen a la primera categoría y los modelos de volatilidad estocástica a la segunda categoríd 1 . Usualmente en la ecuación (25) $\mu_{t}$ tiene un comportamiento de ruido blanco por lo que se trabaja con la serie de los retornos. El modelo para la serie $\mu_{t}$ (si existe) se conoce como la ecuación de la media para $r_{t}$ y el modelo para $\sigma_{t}^{2}$ es la ecuación de la volatilidad para $r_{t}$, de acá en adelante no se modela $a_{t}$ sino $r_{t}$, es decir, no existe un modelo adecuado para la media y se trabaja directamente los retornos que entonces deberán ser ruido blanco.

\subsection{Modelos de volatilidad estocástica}

Los modelos de volatilidad estocástica son parecidos a los GARCH, pero ellos añaden un término de ruido estocástico a la ecuación para $\sigma_{t}$. Un modelo GARCH $(1,1)$ para un retorno, el cual se denota acá como $r_{t}$ está dado por:

$$
\begin{gathered}
r_{t}=\sigma_{t} \epsilon_{t} \\
\sigma_{t}^{2}=\alpha_{0}+\alpha_{1} r_{t-1}^{2}+\beta_{1} r_{t-1}^{2}
\end{gathered}
$$

donde $\epsilon_{t}$ es un ruido blanco gaussiano. Si se define

$$
h_{t}=\log \sigma_{t}^{2} \text { y } y_{t}=\log r_{t}^{2},
$$

entonces, (30) puede ser escrito como:

$$
y_{t}=h_{t}+\log \epsilon_{t}^{2}
$$

\footnotetext{
${ }^{1}$ A propósito del marco teórico de los modelos GARCH, ver Tsay (2002) y Zea \& Rodríguez (2007) secciones 3.1 y 3.2 .
} 
La ecuación (32) es considerada la ecuación de observación y la varianza estocástica $h_{t}$ es considerada un proceso de estado no observado. Similar a (31), el proceso de volatilidad sigue, en su forma básica, una autorregresión de primer orden,

$$
h_{t}=\phi_{0}+\phi_{1} h_{t-1}+w_{t},
$$

donde $w_{t}$ es un ruido blanco gaussiano y su varianza es $\sigma_{w}$.

Juntas las ecuaciones (32) y (33) conforman el modelo de volatilidad estocástica autorregresiva de Primer orden, debido a Harvey et al. (1994). Si $\epsilon_{t}^{2}$ tiene una distribución log-normal, (30) y (31) formarían un modelo de estados gaussiano y se podrían usar los resultados convencionales de los modelos de regresión dinámica para ajustar el modelo a los datos. Infortunadamente, $y_{t}=\log r_{t}^{2}$ raramente se distribuye como una normal, así que si se mantiene el supuesto de normalidad de $\epsilon_{t}$, entonces $\log \epsilon_{t}^{2}$ está distribuida como el logaritmo de una variable ji-cuadrado con un grado de libertad. Esta densidad está dada por:

$$
f x=\frac{1}{\sqrt{2 \pi}} \exp \left\{-\frac{1}{2}\left(e^{x}-x\right)\right\}-\infty<x<\infty
$$

La media y varianza de esta distribución son -1.27 y $\pi^{2} / 2$, respectivamente, la densidad (34) es altamente sesgada con una cola larga a la derecha. Varios enfoques para ajustar un modelo de volatilidad estocástica han sido examinados, estos métodos incluyen una amplia variedad de supuestos sobre los procesos de ruido. Existen varias clases de métodos tanto bayesianos, como no bayesianos, así como métodos de simulación para la inferencia clásica. Kim et al. (1998) proponen modelar el logaritmo de una variable aleatoria ji-cuadrado mediante la mezcla de siete normales para aproximar los primeros cuatro momentos a la distribución del error observacional. Sin embargo, en un esfuerzo por hacer las cosas más simples se re-escribirá la ecuación de observación (32), y con $y_{t}=\ln r_{t}^{2}$ como:

$$
y_{t}=\alpha+h_{t}+\eta_{t}
$$

donde $\eta_{t}$ es ruido blanco y su distribución es la mezcla de dos normales una de ellas centrada en cero. En particular, escribiendo

$$
\eta_{t}=u_{t} z_{t o}+\left(1-u_{t}\right) z_{t o}
$$

donde $u_{t}$ es un proceso independiente e idénticamente distribuido, $\operatorname{Pr}\left\{u_{t}=0\right\}=$ $\pi_{0}, \operatorname{Pr}\left\{u_{t}=1\right\}=\pi_{1}\left(\pi_{0}+\pi_{1}=1\right), z_{t o} \sim \operatorname{iid} N\left(0, \sigma_{0}^{2}\right)$, y $z_{t 1} \sim \operatorname{iid} N\left(\mu_{1}, \sigma_{1}^{2}\right)$.

La ventaja de este modelo es que es fácil de ajustar porque usa el supuesto de normalidad. El modelo presentado por las ecuaciones (33), (35) y (36) son similares a las presentadas por Peña \& Guttman (1988) quienes usan la idea de obtener un filtro de Kalman robusto, planteamiento análogo en Kim et al. (1998). Usando los resultados para el modelo lineal con Switching obtenemos las ecuaciones de filtro para esta representación:

$$
h_{t+1}^{t}=\phi_{0}+\phi_{1} h_{t}^{t-1}+\sum_{j=0}^{1} \pi_{t j} K_{t j} \epsilon_{t j}
$$




$$
\begin{gathered}
P_{t+1}^{t}=\phi_{1}^{2} P_{t}^{t-1}+\sigma_{w}^{2}-\sum_{j=0}^{1} \pi_{t j} K_{t j}^{2} \Sigma_{t j} \\
\epsilon_{t 0}=y_{t}-\alpha-h_{t}^{t-1} \\
\epsilon_{t 1}=y_{t}-\alpha-h_{t}^{t-1}-\mu_{1} \\
\Sigma_{t 0}=P_{t}^{t-1}+\sigma_{0}^{2} \\
\Sigma_{t 1}=P_{t}^{t-1}+\sigma_{1}^{2} \\
K_{t 0}=\phi_{1} P_{t}^{t-1} / \Sigma_{t 0} \\
K_{t 1}=\phi_{1} P_{t}^{t-1} / \Sigma_{t 1}
\end{gathered}
$$

donde $h_{t+1}^{t}$ denota el pronóstico de $h_{t+1}$ usando información hasta $t$.

Para completar el filtro se deben conjeturar las probabilidades $\pi_{t 1}=\operatorname{Pr}\left(u_{t}=\right.$ $\left.1 / y_{1}, \ldots, y_{t} F A C\right)$, para $t=1, \ldots, n$; por supuesto, $\pi_{t 0}=1-\pi_{t 1}$. Sea $f_{j}(t / t-1)$ denotar la densidad condicional de $y_{t}$ dado el pasado $y_{1}, \ldots, y_{t-1}, \mathrm{y} u_{t}=j(j=0,1)$. Luego,

$$
\pi_{t 1}=\frac{\pi_{1} f_{1}(t / t-1)}{\pi_{0} f_{0}(t / t-1)+\pi_{1} f_{1}(t / t-1)}
$$

donde se asume que la distribución $\pi_{j}$, para $j=0,1$ ha sido especificada a priori. $\mathrm{Si}$ el investigador no tiene ninguna razón para preferir un estado sobre otro $\pi_{0}=$ $\pi_{1}=0.5$ será suficiente. Infortunadamente, obtener una expresión exacta para los valores de $f_{j}(t / t-1)$, es difícil; aunque se puede dar una expresión explícita de $f_{j}(t / t-1)$, la computación de la densidad es prohibitiva. Una aproximación viable es escoger que $f_{j}(t / t-1)$ sea una densidad normal, $N\left(h_{t}^{t-1}+\mu_{j}, \Sigma_{t j}\right)$

\section{Valor en riesgo}

En la actualidad se dispone de varias aproximaciones para cuantificar el riesgo de mercado, tales como simulación histórica, simulación tipo Monte Carlo, método "delta normal", modelos de duración, análisis de sensibilidad, las pruebas de valor extremo (stress testing), y otras (De Lara 2002). En particular, en los años recientes, el Valor en Riesgo (VeR) se ha convertido en una de las técnicas más populares en las industrias bancaria, aseguradora y financiera para estimar el riesgo de mercado de sus inversiones. Lo anterior es explicado por que el VeR provee una respuesta simple a la pregunta de, ¿cuál es la pérdida financiera de un portafolio predicha (con probabilidad $\alpha$ ) sobre un horizonte de tiempo dado? La respuesta es el VeR a un nivel $\alpha$, que además tiene la ventaja de estar expresado en la moneda en que está el activo y es fácilmente interpretable. El VeR está definido como el valor del portafolio que podría perderse durante fluctuaciones adversas y severas del mercado. En otras palabras, el VeR al nivel $\alpha$, significa que en un tiempo determinado la máxima pérdida potencial de un activo o portafolio de activos no excederá el VeR a un nivel de confiabilidad 2 del $(1-\alpha)$.

\footnotetext{
${ }^{2}$ Para cálculos de los VeR de activos individuales y en portafolios compuestos de mas de un activo consultar Jorion (2000) o De Lara (2002).
} 
En el presente trabajo se emplearán los VeR de un mercado cambiario para horizontes cortos, siguiendo las recomendaciones del comité de Basilea en 1996, se consideran tenencias de un solo día. Específicamente se modelan los VeR usando modelos de volatilidad estocástica autorregresivos, como los descritos antes, pues estos permiten modelar comportamientos de persistencia en el largo plazo y asimetrías de la volatilidad, más adecuadamente que los $\mathrm{GARCH}^{3}$.

Para evaluar y calibrar los modelos de medición de riesgo, el comité de Basilea y el grupo de los treinta (G-30) recomiendan realizar una prueba conocida como backtesting. En esta prueba es necesario comparar el valor en riesgo calculado con las pérdidas y/o ganancias reales.

\section{Resultados}

Como ya se ha mencionado, las variables de estudio fueron tasa de cambio Peso/Dólar, Euro/Dólar, Libra/Dólar y Yen/Dólar; las unidades de la primera se expresan en Pesos colombianos por Dólar, la segunda en Euros por Dólar, la tercera en Libras esterlinas por Dólar y la tercera en Yenes por Dólar. Corresponden a series diarias desde el 3 de enero de 2000 al 11 de mayo de 2007, resultando un total de 1744 observaciones por cada serie, al tener en cuenta solo los días hábiles en todos estos mercados. Los resultados acá presentados fueron obtenidos mediante el paquete estadístico R (R Development Core Team 2007, Trapletti \& Hornik 2007). Se observa que la serie de cada una de las tasas de cambio (figura 1) no son estacionarias ni en media ni en varianza, las series Euro/Dólar y Libra/Dólar mantienen una tendencia creciente aunque existen muchas fluctuaciones, la serie Peso/Dólar mantuvo por su parte durante mucho tiempo una tendencia creciente pero en los últimos tiempos se viene observando una aguda devaluación, mientras que la serie Yen/Dólar ha presentado por su parte un comportamiento más errático.

Para analizar adecuadamente esta serie se tomaron los retornos simples (no continuos) de cada una de las series, por ejemplo, para el Yen se calculó de la siguiente manera, $r_{t}=100 * \frac{x_{t}-x_{t-1}}{x_{t-1}}$, donde $x_{t}$ es la tasa de cambio Yen a Dólar, $r_{t}$ se conoce como retorno porcentual y se trabaja como procedimiento usual cuando se analizan series de tiempo financieras. A las tasas de cambio de Peso/Dólar, Euro/Dólar, Libra/Dólar y Yen/Dólar (figura 1) se les calculó sus retornos (figura $2 \longdiv { 4 }$.

Las series de cada uno de los retornos tienen media muy cercana a cero y la desviación estándar es de una magnitud considerable respecto a la media; la serie Yen tiene una asimetría negativa mucho mayor a la de las otras series de retornos las cuales están muy cerca de la simetría.

\footnotetext{
${ }^{3}$ Para ver más detalles de como se confrontan estas dos metodologías véase Carnero et al. (2004).

${ }^{4}$ De ahora en adelante se denota la serie de los retornos como Peso, Euro, Libra y Yen, respectivamente.
} 

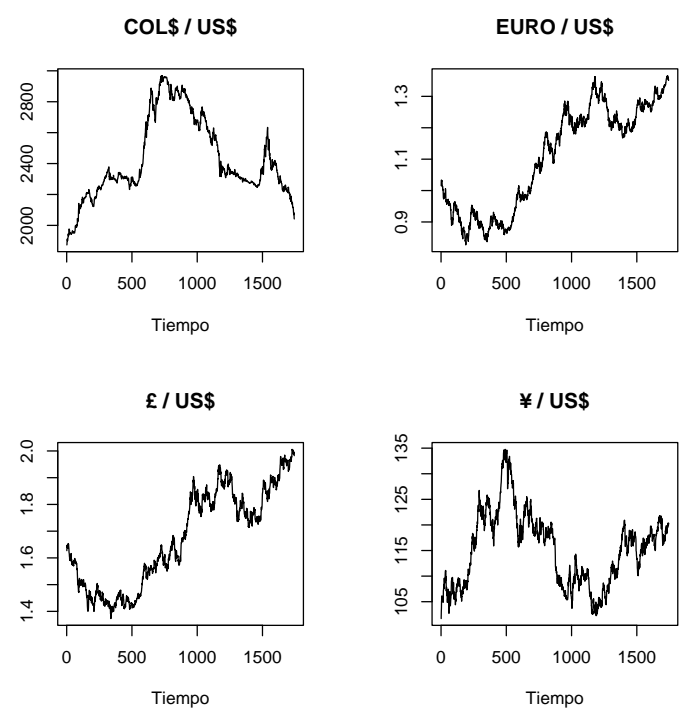

Figura 1: Tasa de cambio a dólar

Los retornos presentan una alta curtosis (leptocúrtica), especialmente los retornos del Peso (tabla 6, Apéndice). Esto último indica la posible presencia de algunos datos atípicos o de colas pesadas.

Tabla 1: Estadísticas descriptivas de los retornos

\begin{tabular}{lrrrrrrrrrr}
\hline \hline & $\mathrm{n}$ & Min. & 1st Qu. & Median & 3rd Qu. & Max. & Sd & Var & Skew. & Kurt. \\
\hline Peso & 1743 & -3.04 & -0.21 & -0.01 & 0.18 & 2.80 & 0.45 & 0.20 & 0.05 & 9.01 \\
Euro & 1743 & -3.04 & -0.21 & -0.01 & 0.18 & 2.80 & 0.63 & 0.39 & -0.04 & 3.64 \\
Libra & 1743 & -2.16 & -0.31 & 0.01 & 0.32 & 2.10 & 0.53 & 0.29 & -0.04 & 3.58 \\
Yen & 1743 & -2.90 & -0.34 & 0.01 & 0.35 & 2.47 & 0.60 & 0.36 & -0.26 & 4.53 \\
\hline
\end{tabular}

Al observar la figura 2 se concluye que los retornos presentan algunos periodos de agrupamientos de volatilidad, es decir, hay momentos en donde el comportamiento de la serie es muy fluctuante y otros en donde se observa mayor calma; estos agrupamientos son mucho más notorios en la serie Peso. En cuanto a la distribución de probabilidad que ajustan los retornos se evidencia con la prueba de Shapiro-Wilk (tabla 2) que ninguna de estas series muestra evidencia de seguir una distribución gaussiana.

Sin embargo, al observar el histograma (figura 4), el QQ-plot (figura 3) y detallar las medidas de asimetría y curtosis, estas no presentan una magnitud muy alejada a la de la distribución normal, a excepción de la serie Peso, la cual es claramente leptocúrtica. En las series Euro, Libra y Yen no se observa una desviación al supuesto gaussiano demasiado notoria. 

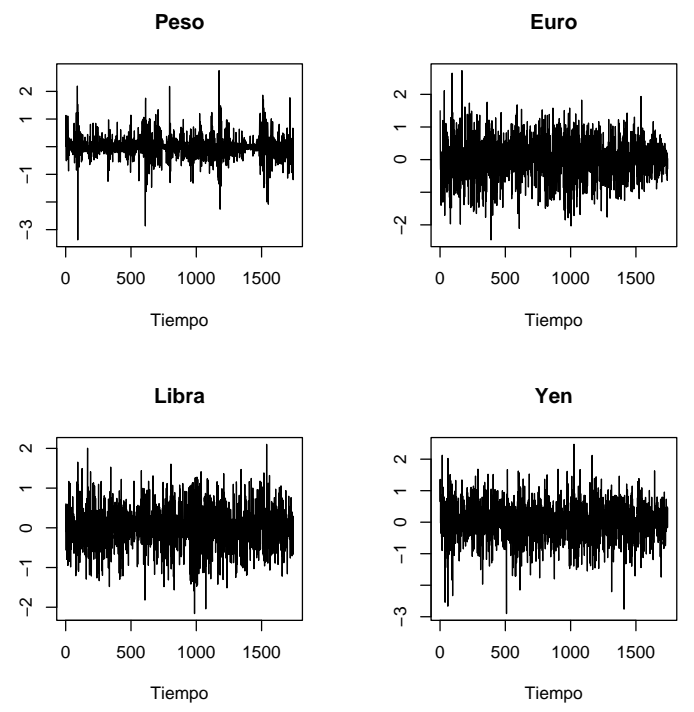

Figura 2: Retornos $\left(r_{t}\right)$
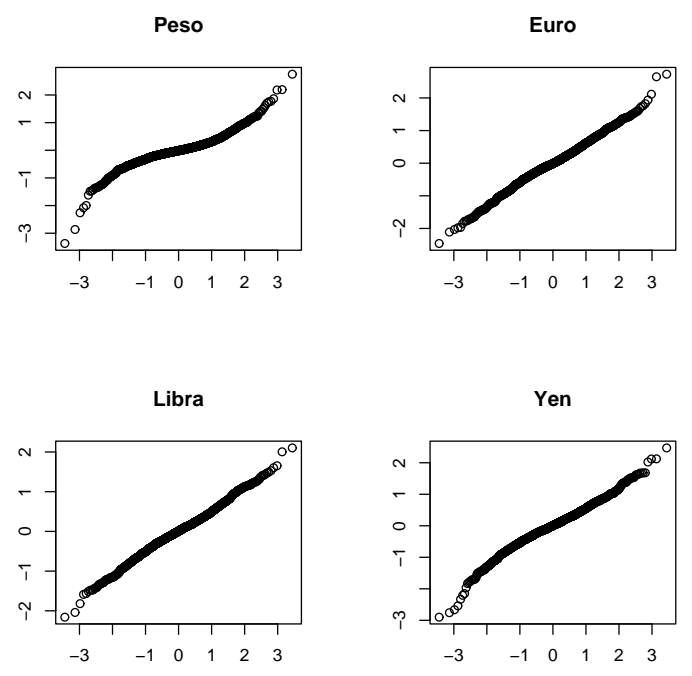

Figura 3: QQ-plot de los retornos 

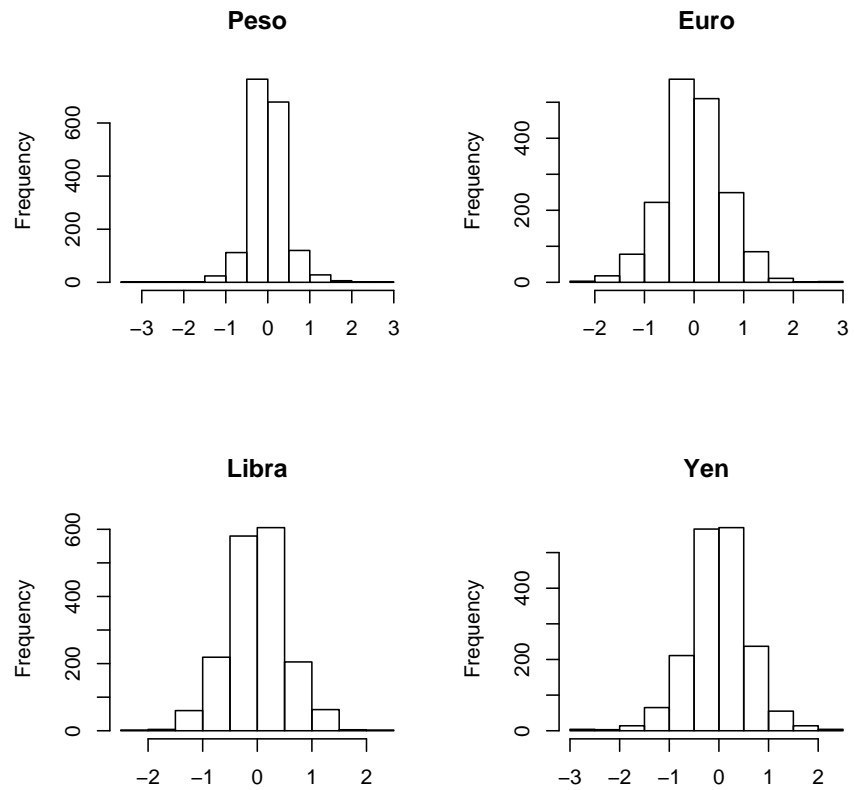

Figura 4: Histograma de los retornos

Tabla 2: Estadístico de Shapiro-Wilk para la serie de los retornos

\begin{tabular}{ccc}
\hline \hline Peso & $\mathrm{W}$ & $\mathrm{p}$-valor \\
\hline Peso & 0.9252 & 0 \\
Euro & 0.9951 & $1.609 \mathrm{e}-05$ \\
Libra & 0.9952 & $2.251 \mathrm{e}-05$ \\
Yen & 0.9856 & $3.307 \mathrm{e}-12$ \\
\hline
\end{tabular}

Al observar las ACF (función de autocorrelación simple)y PACF (función de autocorrelación parcial) (figura 6 y figura 8) se evidencia que las series de los retornos de Euro, Libra y Yen se comportan aproximadamente como ruido blanco y aunque existen unas pocas autocorrelaciones simples y parciales distintas a cero, la ausencia de autocorrelaciones significativas, se corrobora con la prueba de Ljung-Box, la cual examina la hipótesis de no autocorrelación con un determinado número de rezagos o como se conoce en la literatura anglosajona, lags (véase tabla 7). En la prueba se observa que no se rechaza el supuesto de no autocorrelación para ninguna de estas tres series mencionadas. Por su parte, la serie de retornos Peso, presenta autocorrelaciones simples con un ligero patrón de decaimiento y en la figura 5 se observa que las tres primeras autocorrelaciones parciales son distintas de cero y de ahí en adelante las autocorrelaciones parciales no son significativas. 
Lo anterior muestra evidencia de un posible modelo autorregresivo de orden 3 $(\mathrm{AR}(3))$. Para identificar de una manera más adecuada los modelos para el nivel de cada una de las series de los retornos, se ajustó el modelo que presentó menor criterio de información de Akaike (AIC), los modelos elegidos coinciden con los vislumbrados con las herramientas de la ACF y la PACF. Con la prueba de Ljung-Box se evaluaron las autocorrelaciones del cuadrados de los retornos (no se presentan acá para mantener la brevedad del documento), las conclusiones respecto al GARCH a utilizar son similares a las obtenidas mediante la evidencia gráfica de las ACF y PACF. En cuanto a la verificación de supuestos mediante los residuos estandarizados, se observa que estos presentan comportamiento de ruido blanco, media cero y varianza cercana a uno, sin embargo presenta niveles altos de curtosis (curtosis que en algunos de los residuos llegan a ser mayores a 9), lo cual hace pensar que sería necesario ajustar los retornos a una distribución distinta a la gaussiana.

ACF Peso

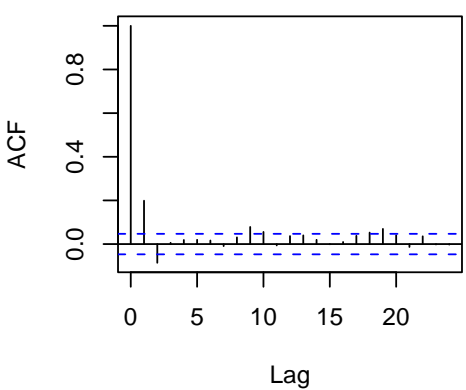

ACF residual Peso

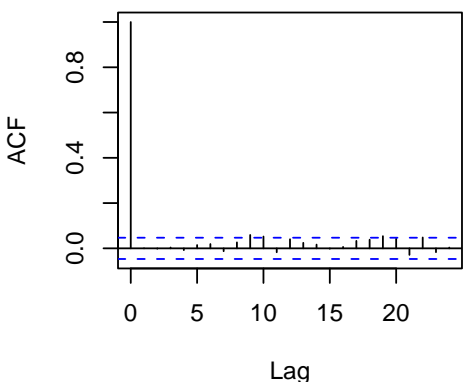

PACF Peso

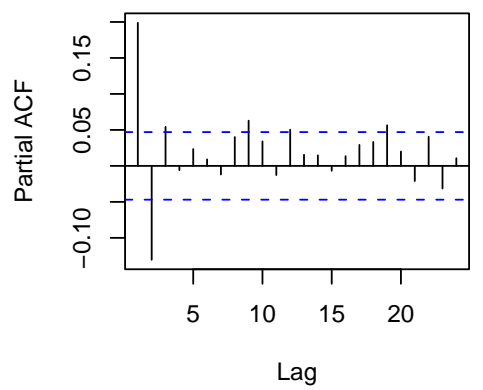

PACF residual Peso

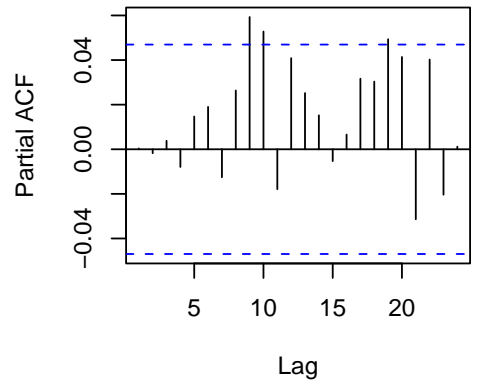

Figura 5: $A C F$ y PACF retornos Peso 

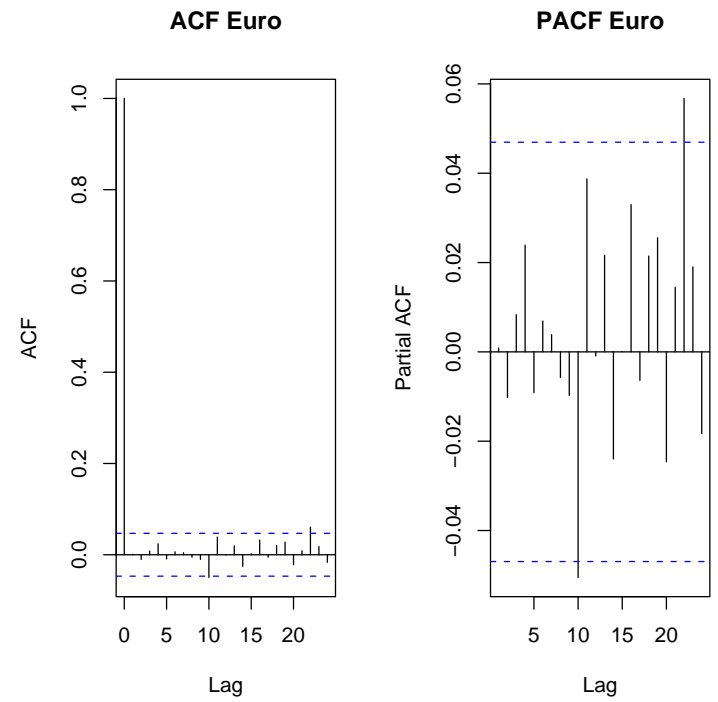

Figura 6: $A C F$ y PACF retornos Euro
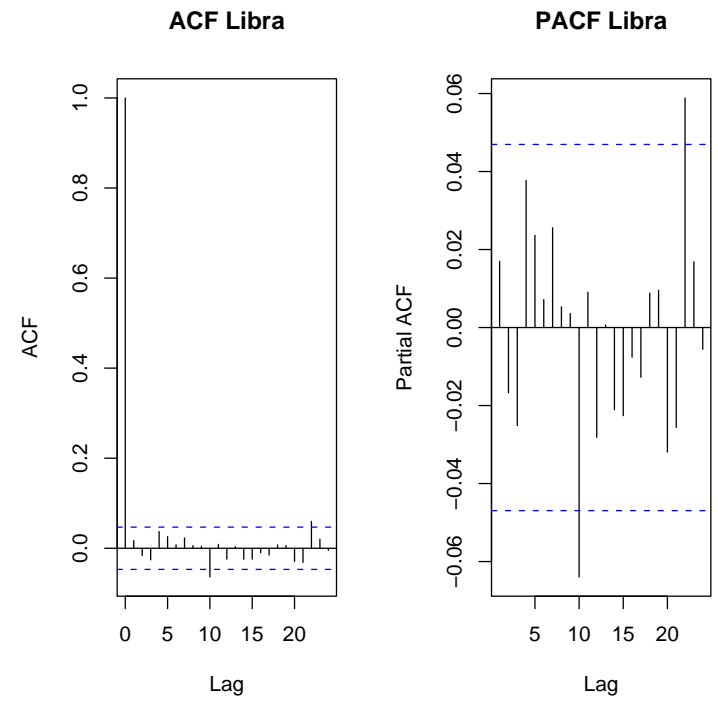

Figura 7: $A C F$ y PACF retornos Libra 

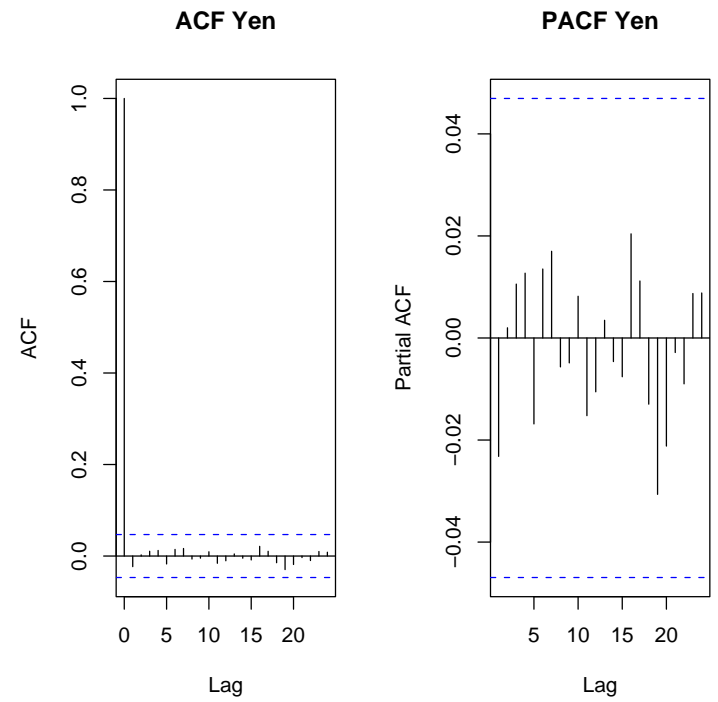

Figura 8: $A C F$ y PACF retornos Yen

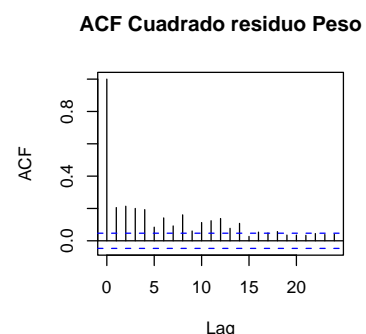

PACF Cuadrado residuo Peso

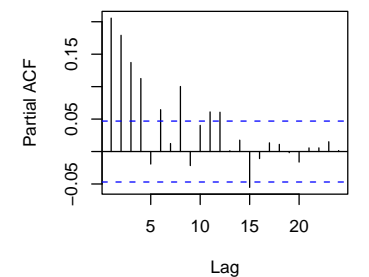

ACF cuadrado Euro

PACF cuadrado Euro
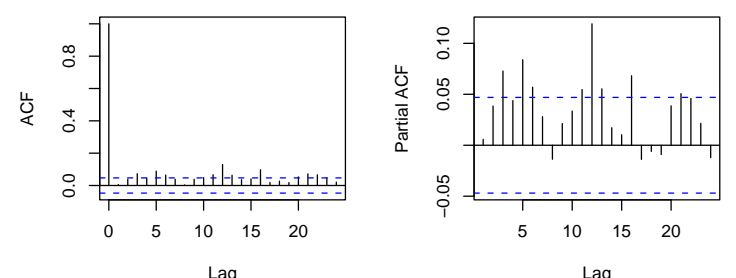

Figura 9: ACF y PACF de los cuadrados de los retornos de Peso y Euro 


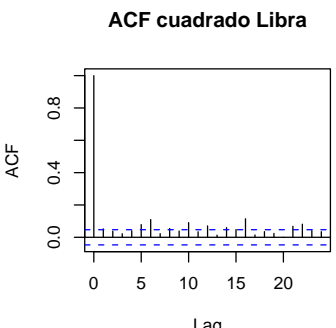

Lag

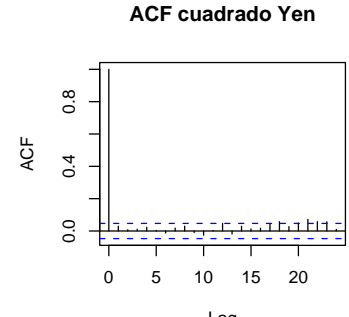

Lag

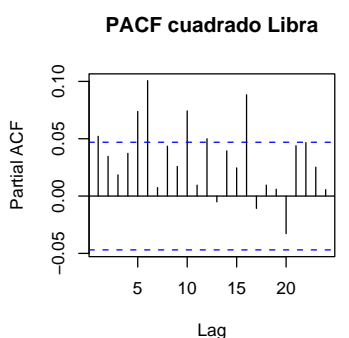

PACF cuadrado Yen

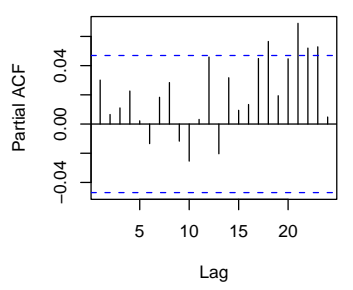

Figura 10: ACF y PACF de los cuadrados de los retornos de Libra y Yen

Una vez establecido un modelo para los niveles de los retornos (a la derecha), la idea es encontrar un modelo para la varianza condicional de los residuos; sin embargo, las series Euro, Libra y Yen se modelan directamente, luego de restar la media a cada serie, ya que tenían un comportamiento de ruido blanco. A la serie Peso se le ajusta un $\mathrm{AR}(3)$ y los residuos del modelo se conservan para posteriormente modelarlos. La serie de los cuadrados de Euro, Libra y Yen permite tener evidencia de cuáles son los modelos GARCH más adecuados para cada una de ellas.

En la figuras 9 a la 10 se observan las autocorrelaciones parciales de los retornos al cuadrado, estas son distintas de cero en rezagos bastante elevados, lo cual significa que, de especificar algún modelo AR puro, este tendría un orden bastante alto; un modelo GARCH evita la necesidad de tener que especificar modelos ARCH de una magnitud tan grande. Después de elegir el modelo usando el AIC, los modelos ajustados fueron $\mathrm{AR}(3)-\mathrm{ARCH}(2)$ para Peso, y GARCH(1,1) para Euro, Libra y Yen.

Se observa que para las series Euro, Libra y Yen, la magnitud de $\alpha_{1}+\beta_{1}<1$ lo que significa reversión a la media de la volatilidad y volatilidades implicadas cercanas a 1 . Note que en Euro $\alpha_{1}+\beta_{1} \approx 1$ lo que muestra una gran persistencia en la volatilidad. El intercepto $\alpha_{0}$ no es significativo en Euro, por su parte en Libra y en Yen, si bien $\alpha_{0}$ es significativo, es de una magnitud muy pequeña. Para los residuos de Peso no se nota una gran persistencia de la volatilidad. 
A continuación se observa los resultados de la estimación del modelo GARCH para las cuatro series.

Tabla 3: ARCH(2) para Peso

\begin{tabular}{rrrrr}
\hline \hline Coef. & Estimación & Error Est. & Estad. t & P-Valor \\
\hline$\alpha_{0}$ & 0.07184 & 0.002477 & 29.01 & 0 \\
$\alpha_{1}$ & 0.41875 & 0.025948 & 16.14 & 0 \\
$\alpha_{2}$ & 0.33420 & 0.026529 & 12.60 & 0 \\
\hline
\end{tabular}

Nótese que para los retornos del peso se trabajaron con los residuos de un modelo $\mathrm{AR}(3)$ y a estos se les ajustó un $\mathrm{ARCH}(2)$.

Tabla 4: $\operatorname{GARCH}(1,1)$ para Euro

\begin{tabular}{rrrrr}
\hline \hline Coef. & Estimación & Error Est. & Estad. t & P-Valor \\
\hline$\alpha_{0}$ & $5.47 \mathrm{E}-15$ & 0.00079 & 0.00 & 1 \\
$\alpha_{1}$ & 0.02752 & 0.0056 & 4.78 & 0 \\
$\beta_{1}$ & 0.9721 & 0.00628 & 154.82 & 0 \\
\hline
\end{tabular}

Tabla 5: $\operatorname{GARCH}(1,1)$ para Libra

\begin{tabular}{rrrrr}
\hline \hline Coef. & Estimación & Error Est. & Estad. t & P-Valor \\
\hline$\alpha_{0}$ & 0.00726 & $2.89 \mathrm{E}-03$ & 2.51 & 0.012 \\
$\alpha_{1}$ & 0.03952 & 0.009456 & 4.18 & 0.000 \\
$\beta_{1}$ & 0.93510 & 0.016937 & 55.206 & 0.000 \\
\hline
\end{tabular}

Tabla 6: GARCH(1,1) para Yen

\begin{tabular}{rrrrr}
\hline \hline Coef. & Estimación & Error Est. & Estad. t & P-Valor \\
\hline$\alpha_{0}$ & 0.007731 & 0.00429 & 1.80 & 0.072 \\
$\alpha_{1}$ & 0.016497 & 0.00573 & 2.88 & 0.004 \\
$\beta_{1}$ & 0.961539 & 0.01661 & 57.91 & 0.000 \\
\hline
\end{tabular}

A continuación se procede a trabajar con un modelo de volatilidad estocástica como alternativa al GARCH ya ajustado. Como procedimiento usual para los modelos de volatilidad estocástica se trabaja con las series: $y_{t}=\log r_{t}^{2}$ y $h_{t}=\log \sigma_{t}^{2}$. Como ya se mencionó, el modelo de volatilidad estocástica presenta múltiples ventajas frente a los GARCH, entre ellos el de su gran flexibilidad, puesto que trabaja la serie de la volatilidad como un proceso estocástico no observable y sujeto a error aleatorio. Recordando que el modelo de volatilidad está dado por las ecuaciones (33), (35) y (36) con los supuestos anteriormente descritos, los cuales se reescriben de nuevo:

$$
h_{t}=\phi_{0}+\phi_{1} h_{t-1}+w_{t},
$$

donde $w_{t}$ es ruido blanco gaussiano,

$$
y_{t}=\alpha+h_{t}+\eta_{t},
$$

En donde,

$$
\eta_{t}=u_{t} z_{t o}+\left(1-u_{t}\right) z_{t o},
$$


donde $u_{t}$ es un proceso independiente e idénticamente distribuido, $\operatorname{Pr}\left[u_{t}=0\right]=\pi_{0}$, $\operatorname{Pr}\left[u_{t}=1\right]=\pi_{1}$ Con $\left(\pi_{0}+\pi_{1}=1\right), z_{t 0} \sim N\left(0, \sigma_{0}^{2}\right)$, y $z_{t 1} \sim N\left(\mu_{1}, \sigma_{1}^{2}\right) . \sigma_{w}$ es la varianza de $w_{t}$. Tanto $z_{t 0}$ como $z_{t 1}$ están idénticamente distribuidas.

Las estimaciones de los parámetros del modelo de volatilidad para cada una de las series se presentan a continuación:

Tabla 7: Resultados de estimación del modelo de Volatilidad Estocástica: Peso

\begin{tabular}{lrrr}
\hline \hline coef. & lim. inferior & estimación & lim. superior \\
\hline$\phi_{0}$ & -0.26 & -0.06 & 0.13 \\
$\phi_{1}$ & 0.94 & 0.96 & 0.99 \\
$\sigma_{w}$ & 0.26 & 0.38 & 0.50 \\
$\alpha$ & -6.13 & -0.74 & 4.64 \\
$\sigma_{0}$ & 0.87 & 1.02 & 1.17 \\
$\mu_{1}$ & -2.74 & -2.33 & -1.93 \\
$\sigma_{1}$ & 2.37 & 2.60 & 2.82 \\
\hline
\end{tabular}

Tabla 8: Resultados de estimación del modelo de Volatilidad Estocástica: Euro

\begin{tabular}{lrrr}
\hline \hline coef. & lim. inferior & estimación & lim. superior \\
\hline$\phi_{0}$ & -0.00 & -0.00 & -0.00 \\
$\phi_{1}$ & 1.00 & 1.00 & 1.00 \\
$\sigma_{w}$ & 0.01 & 0.05 & 0.09 \\
$\alpha$ & -1.58 & -1.35 & -1.12 \\
$\sigma_{0}$ & 2.20 & 2.39 & 2.58 \\
$\mu_{1}$ & 2.29 & 2.64 & 2.99 \\
$\sigma_{1}$ & 0.94 & 1.05 & 1.15 \\
\hline
\end{tabular}

Tabla 9: Resultados de estimación del modelo de Volatilidad Estocástica: Libra

\begin{tabular}{lrrr}
\hline \hline coef. & lim. inferior & estimación & lim. superior \\
\hline$\phi_{0}$ & -0.06 & 0.00 & 0.06 \\
$\phi_{1}$ & 0.94 & 0.98 & 1.01 \\
$\sigma_{w}$ & 0.03 & 0.11 & 0.18 \\
$\alpha$ & -6.36 & -3.98 & -1.60 \\
$\sigma_{0}$ & 2.31 & 2.52 & 2.73 \\
$\mu_{1}$ & 2.00 & 2.39 & 2.78 \\
$\sigma_{1}$ & 0.97 & 1.09 & 1.22 \\
\hline
\end{tabular}

Tabla 10: Resultados de estimación del modelo de Volatilidad Estocástica: Yen

\begin{tabular}{lrrr}
\hline \hline coef. & lim. inferior & estimación & lim. superior \\
\hline$\phi_{0}$ & -1.60 & 2.10 & 5.79 \\
$\phi_{1}$ & -1.07 & -0.95 & -0.84 \\
$\sigma_{w}$ & -0.07 & 0.05 & 0.17 \\
$\alpha$ & -4.37 & -2.43 & -0.48 \\
$\sigma_{0}$ & 1.04 & 1.16 & 1.28 \\
$\mu_{1}$ & -2.75 & -2.36 & -1.96 \\
$\sigma_{1}$ & 2.24 & 2.45 & 2.66 \\
\hline
\end{tabular}

Los parámetros del modelo de volatilidad que tiene una interpretación más directa son $\phi_{0}, \phi_{1}$ y $\sigma_{w}$. Respecto a $\phi_{1}$ y $\phi_{0}$ si analizamos la ecuación de $h_{t}$ como un proceso autorregresivo notamos que tres de las cuatro series son estacionarias, esto se nota analizando las raíces del polinomio característico asociado a los parámetros y verificando si estas están dentro del círculo unitario. Todas las series a excepción de la series del Yen dan cercanas a uno, esto significa que existe un efecto considerable 
del pasado y que este es muy persistente en el tiempo. Para la serie del Yen por tanto sería conveniente hacer una estimación con restricciones para garantizar la estacionariedad del proceso autorregresivo.

Respecto a $\sigma_{w}$ que es la varianza del error asociado a este proceso autorregresivo, notamos que las series de Euro y Yen son las que menor varianza presentan en el residual de la ecuación de la volatilidad, por su parte las series de Libra y Peso presentan una varianza mucho más grande.

Mediante los residuos estandarizados se comprobó que estos tienen un comportamiento muy similar a ruido blanco, pero no cumplen con tener una distribución gaussiana estándar, si bien su media, varianza y asimetría es muy similar a la de una normal. Estos tienen una curtosis mayor a 3, sin embargo, el comportamiento de los residuos estandarizados es ligeramente mejor en el modelo SV que en el GARCH.

En el apéndice en las figuras 11 a la 14 se presenta a $\sigma_{t}$ estimada mediante un modelo GARCH y un modelo de volatilidad estocástica (SV). Esta medida de volatilidad será necesaria para estimar posteriormente el valor en riesgo.

Se observa que el modelo de volatilidad estocástica reproduce de manera mucho más exacta el comportamiento dinámico de los retornos. Se evidenció en otro ejercicio, no presentado en este documento por limitaciones de espacio, que los intervalos para los retornos son mucho más pequeños en un modelo de volatilidad estocástica (SV) que en un GARCH. Los dos modelos reproducen de manera muy similar en cuanto a forma a la volatilidad, a excepción de la serie para Yen, que debido a sus grandes fluctuaciones presenta unos incrementos y descensos muy profundos en su volatilidad. La escala en la que el modelo de volatilidad estocástica predice la volatilidad es distinta a la predicha por los modelos GARCH.

\section{Resultados del análisis retrospectivo Backtesting}

A pesar de las debilidades de la prueba Backtesting, reportadas entre otros por Balzarotti, Del Canto y Delfiner (2000) y mas aún Powell y Balzarotti (1997) en un documento del Banco Interamericano de Desarrollo en el que tratan el tema específicamente para Latinoamérica, los resultados de esta prueba se presentan en la tabla 11.

Tabla 11: Resultados de la prueba Backtesting

\begin{tabular}{rccccc}
\hline \hline Moneda & Modelo & N. Fallas & \% Fallas & Estad. LR & P-Valor \\
\hline Peso & GARCH & 6 & $2.4 \%$ & 3.4 & $6.5 \%$ \\
Peso & SV & 1 & $0.4 \%$ & 1.2 & $26.6 \%$ \\
Euro & GARCH & 0 & $0.0 \%$ &. &.$\%$ \\
Euro & SV & 32 & $12.5 \%$ & 106.6 & $0.0 \%$ \\
Yen & GARCH & 2 & $0.8 \%$ & 0.1 & $71.9 \%$ \\
Yen & SV & 2 & $0.8 \%$ & 0.1 & $71.9 \%$ \\
Libra & GARCH & 6 & $2.4 \%$ & 3.4 & $6.5 \%$ \\
Libra & SV & 3 & $1.2 \%$ & 0.1 & $78.3 \%$ \\
\hline
\end{tabular}


En este trabajo se calculan los pronósticos a horizontes de un día, para ver la metodología y los lineamentos que se utilizan en las instituciones bancarias, el comité de Basilea y la misma Superintendencia Financiera de Colombia, respecto a los pronósticos, ver Grane \& Veiga (2007).

\subsection{Cálculo del VeR}

Como $\epsilon_{t}$ se supone ruido blanco gaussiano, el pronóstico puntual para $r_{t}$ será cero. Se sabe que $\log \left\{\operatorname{Var}\left(r_{t} / Y_{t-1}\right)\right\}=\log \left\{\sigma_{t}^{2}\right\}$, por tanto se puede obtener el error estándar para $r_{t}$ y obtener la cota inferior de su intervalo de confiabilidad como se muestra en la ecuación (46).

Para el cálculo del valor en riesgo, suponiendo que $r_{t}$ sigue una distribución normal con media igual a cero y varianza condicional dada por (2), $\sigma_{t}$, para un horizonte de tiempo de un día y un nivel de confianza del $99 \%$ se calcula así (De Lara 2002).

$$
V e R=C \times\left(\mu_{t+1 / t}-2.326 \sigma_{t+1 / t}^{2}\right)
$$

con $\mathrm{C}$ el valor de la inversión o tamaño del portafolio. Para un horizonte de tiempo k y el mismo nivel de significación es igual a,

$$
V e R=C \times\left(\mu_{t+k / t}-2.326 \sigma_{t+k / t}^{2}\right)
$$

El cálculo del VeR de cada moneda y del portafolio se presentan en la tabla 12 , también se presentan allí las ponderaciones o participación en un portafolio óptimo. Estas participaciones se obtienen de una asignación óptima que minimiza la varianza del portafolio; es decir, arrojan una varianza de portafolio de 0.000636 . El resultado del cálculo del VeR para una inversión de US\$20,000,000 está dado en la tabla 12

Tabla 12: Cálculo del VeR del portafolio

\begin{tabular}{rrrr}
\hline \hline \multicolumn{4}{c}{ MODELO } \\
\hline MONEDA: & GARCH & SV & Partic. \\
Peso & $-\$ 10,883,556$ & $-\$ 12,369,480$ & $32.9 \%$ \\
Euro & $-\$ 3,518,578$ & $-\$ 2,310,403$ & $13.0 \%$ \\
Libra & $-\$ 7,339,949$ & $-\$ 10,290,594$ & $24.2 \%$ \\
Yen & $-\$ 10,129,565$ & $-\$ 17,464,681$ & $29.9 \%$ \\
\hline Portafolio & $-\$ 31,954,530$ & $-\$ 44,321,885$ & $100 \%$ \\
\hline
\end{tabular}

\section{Conclusiones}

En este documento se presentó un ejercicio de cálculo del VeR de portafolio del mercado cambiario usando modelos de volatilidad estocástica, mediante el uso de 
series diarias de tasa de cambio Peso/Dólar, Yen/Dólar, Euro/Dólar y Libra/Dólar.

Los resultados muestran la idoneidad de la alternativa SV, al menos y por ahora frente a la alternativa GARCH. Los resultados de la prueba de backtesting indican que la metodología del uso del modelo SV para cálculo del VeR muestra cumplir los lineamentos del comité de Basilea. En ese caso, de ser adoptado este tipo de técnicas, se espera, como es usual en los ejercicios de pronóstico ex-ante, que una adecuada combinación de al menos estas dos metodologías, arroje mejores resultados que los pronósticos individuales.

Queda pendiente, para futuros trabajos, realizar esta evaluación contra el suavizamiento exponencial usado por RiskMetrics ${ }^{T M}$, y contra otras especificaciones del GARCH o incluso otras alternativas de modelos de volatilidad estocástica como las más convencionales estudiadas en Jacquier et al. (1999) o uno más novedoso como la propuesta de Breidt et al. (1998), la cual considera efectos en volatilidad no solo de corto sino de largo plazo.

Otra alternativa interesante consiste en usar técnicas bootstrapping (Grane \& Veiga 2007) para estimar los intervalos de riesgo y el VeR, en cuyo caso, se puede relajar el supuesto de normalidad e incluso el de simetría en la distribución de los términos de error, así como modelar la dependencia de estos. Además, el extender la aplicación a SV multivariantes (Jacquier et al. 1999), lo cual permitiría estimar las correlaciones entre las monedas o activos incluidos en el portafolio.

Sobra decir que esta metodología es fácilmente extensible para incluir las nueve monedas o más como lo considera la superfinanciera e incluso extenderse para modelar y evaluar riesgo de tasa de interés y otros mercados.

\section{Recibido: 25 de febrero de 2012 Aceptado: 8 de abril de 2012}

\section{Referencias}

Breidt, F., Crato, G. \& de Lima, P. (1998), 'On the detection and Estimation of Long Memory in Stochastic Volatility', Journal of Econometrics, 2, 319-342.

Carnero, M. A., Peña, D. \& Ruiz, E. (2004), 'Persistence and Kurtosis in GARCH and Stochastic Volatility Models', Journal of Financial Econometrics, 2(2), 319-342.

De Lara, A. (2002), Medición y control de riesgos Financieros, Ed. Limusa S. A., México D.F.

Grane, A. \& Veiga, H. (2007), 'Volatility Modelling and Accurate Minimum Capital Risk Requirements. Technical Report 07-47.', Departamento de Estadística, Universidad Carlos III .

Hamilton, J. D. (1989), 'A new approach to the economic analysis of nonstationary time series and the business cycle', Econometrica. 57, 357-384. 
Harvey, A., Ruiz, E. \& Shephard, N. (1994), 'Multivariate Stochastic Variance Models', Review of Economic Studies, 65, 361-393.

Jacquier, E., Polson, N. G. \& Rossi, P. E. (1999), Stochastic Volatility: Univariate and Multivariate Extensions, Technical Report September, University of Chicago, Graduate School of Business.

Jorion, P. (2000), Valor en Riesgo, Limusa, México.

Kim, S., Shephard, N. \& Chib, S. (1998), 'Stochastic Volatility: Likelihood Inference and Comparison with ARCH Models', Review of Economic Studies, 65, 361-393.

McCulloch, R. E. \& Tsay, R. S. (1993), 'Robust Test for the Equality of Variantes', Journal of the American Statistical Association, 88, 968-978.

Peña, D. \& Guttman, I. (1988), A Bayesian approach to robustifying the Kalman filter. In Bayesian Analysis of Time Series and Dynamic Linear Models, J.C. Spall, ed., New York: Marcel Dekker., pp. 227-254.

R Development Core Team (2007), R: A Language and Environment for Statistical Computing, R Foundation for Statistical Computing, Vienna, Austria. ISBN 3-900051-07-0.

*http://www.R-project.org

Shumway, R. \& Stoffer, D. (2006), Time Series Analysis and Its Applications With $R$ Examples, 2 edn, Springer, New York.

Trapletti, A. \& Hornik, K. (2007), tseries: Time Series Analysis and Computational Finance. R package version 0.10-11.

*http://CRAN.R-project.org/

Tsay, R. (2002), Analysis of Financial Time Series, Wiley, New York.

Zea, J. \& Rodríguez, N. (2007), XVII Simposio colombiano de estadística, estadística en la evaluación de riesgos, Cali. 


\section{A. Apéndice}

Tabla 13: Test de Ljung-Box de autocorrelaciones de los retornos

\begin{tabular}{lccccc}
\hline \hline Serie & Estadístico & p-valor & Serie (rezago) & Estadístico & p-valor \\
\hline Retorno Peso & 69.00 & 0.00 & Retorno Peso (10 lag) & 101.78 & 0.00 \\
Retorno Peso & 126.72 & 0.00 & Retorno Peso (50 lag) & 146.57 & 0.00 \\
Retorno Peso & 203.74 & 0.00 & Retorno Euro (1 lag) & 0.00 & 0.97 \\
Retorno Euro & 6.18 & 0.80 & Retorno Euro (20 lag) & 15.39 & 0.75 \\
Retorno Euro & 46.82 & 0.60 & Retorno Euro (100 lag) & 90.00 & 0.75 \\
Retorno Libra & 0.50 & 0.48 & Retorno Libra (10 lag) & 13.98 & 0.17 \\
Retorno Libra & 19.48 & 0.49 & Retorno Libra (50 lag) & 47.10 & 0.59 \\
Retorno Libra g & 100.71 & 0.46 & Retorno Yen (1 lag) & 0.94 & 0.33 \\
Retorno Yen & 3.04 & 0.98 & Retorno Yen (20 lag) & 7.38 & 1.00 \\
Retorno Yen & 44.06 & 0.71 & Retorno Yen (100 lag) & 82.63 & 0.90 \\
\hline
\end{tabular}
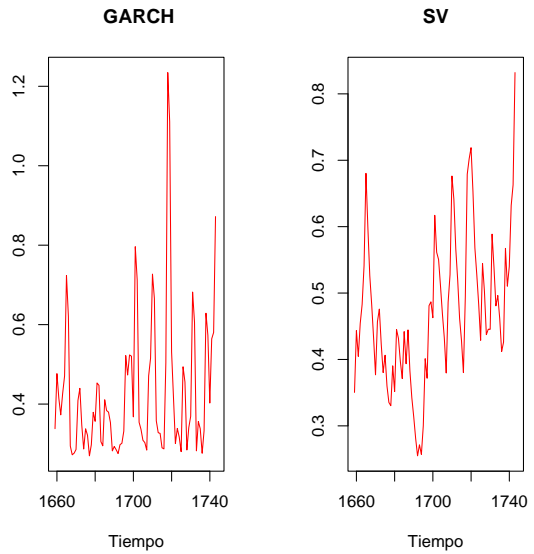

Figura 11: Estimación de $\sigma_{t}$ mediante el uso de un modelo $\operatorname{GARH}(1,1)$ y uno de Volatilidad estocástica para el Peso 

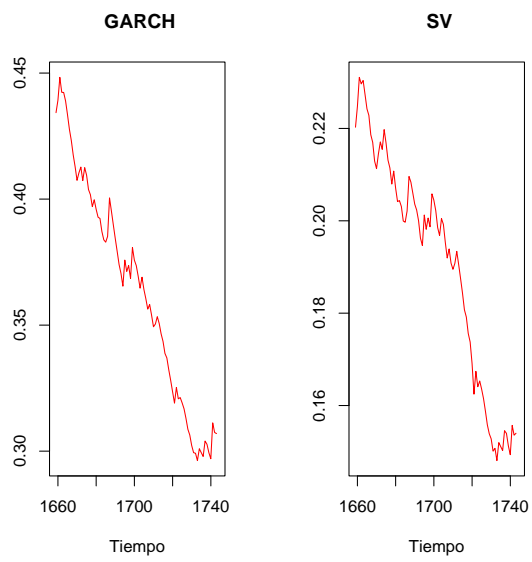

Figura 12: Estimación de $\sigma_{t}$ mediante el uso de un modelo $\operatorname{GARCH}(1,1)$ y uno de Volatilidad estocástica para el Euro
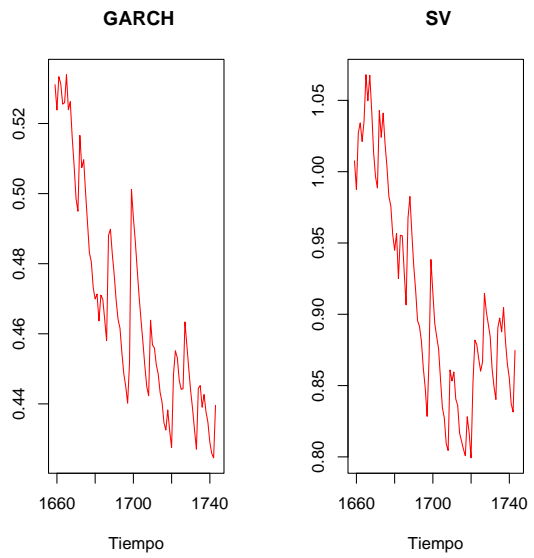

Figura 13: Estimación de $\sigma_{t}$ mediante el uso de un modelo $\operatorname{GARH}(1,1)$ y uno de Volatilidad estocástica para la Libra 